\title{
Mobi-Space - ein modulares, wiederverwendbares Bausystem aus Holz
}

Das Bausystem Mobi-Space wurde zunächst als Mobi-Sku:I entwickelt, um während Grundsanierungen von Schulen den Gemeinden einen ansprechenden Ersatzbau mit hohen energetischen und räumlichen Qualitäten anzubieten. Die wesentlichen Anforderungen an das System sind schnelle Errichtung, gute Demontier- und Wiederverwendbarkeit, ein energetischer Standard der Hülle oberhalb des EnEV-Niveaus und in den bis zu dreigeschossigen Gebäuden ein den regulatorischen Anforderungen entsprechender Schall- und Brandschutz. Das System besteht aus $54 \mathrm{~m}^{2}$ großen Raummodulen, die überwiegend aus hoch vorgefertigten Holztafelelementen bestehen. Eine besondere Herausforderung stellt mit Blick auf eine mehrfache Montage und Demontage die Sicherstellung der horizontalen Lastabtragung und räumlichen Aussteifung dar. Dazu wurden zum Teil Steckverbindungen und Verspannungen, ergänzt durch lösbare Verschraubungen, verwendet. Temporäre Gründungen ergänzen das Gesamtkonzept.

Durch die modulare Konzeption können die Erschließungskonzepte den jeweiligen örtlichen Randbedingungen angepasst werden. Die Strom- und Medienversorgung erfolgt über Steckverbindungen von Modul zu Modul.

Das Bausystem wurde bisher nicht nur als temporärer Schulersatzbau, sondern auch als Mobi-Office, z. B. an der TU Darmstadt, umgesetzt. Insgesamt wurden bisher 25 Projekte realisiert, drei davon bereits demontiert und neu aufgebaut. Das Mobi-Space-Konzept zeigt, wie modulares, industrielles Bauen mit hoher architektonischer Qualität realisiert werden kann.

Keywords Raummodule in Holztafelbauweise; Wiederverwendbarkeit; Bauen, serielles; Bauten, temporäre; Energieeffizienz; TGA; Brandschutz

\begin{abstract}
Modulares und Industrielles Bauen: Eine Systematik industriellen Bauens, umgesetzt durch Raum- und Flächenmodule
\end{abstract}

Das modulare Bauen wird gerade einmal wieder in der gesamten Baubranche sehr intensiv diskutiert. Schnell benötigter zusätzlicher Wohnraum, Raumnot an den Universitäten durch rasant steigende Studierendenzahlen oder temporäre Ausweichquartiere bei grundlegenden Sanierungsarbeiten im Bestand sind beispielsweise Treiber für die Diskussion. Meist sind damit weitere Motive verbunden: Kosten- und Terminsicherheit, schnelle Verfügbarkeit, industrielle, qualitätsgesicherte Fertigung inklusive der technischen Gebäudeausrüstung (TGA), Umgehung der zu koordinierenden Gewerkevielfalt vor Ort, Ausgleich von Fachkräftemangel, Preisvorteile.

\begin{abstract}
Mobi-Space - a modular, re-usable timber construction system

The construction system Mobi-Space was originally developed as Mobi-Sku:I to offer school communities an attractive replacement building with high energetic and spatial quality during renovation periods of their school facilities. The essential requirements are a short erection time, ability of non-destructive demolition, an energetic standard above regulations and an adequat noise- and fire protection. The system exists out of $54 \mathrm{~m}^{2}$ big modules, created from highly prefabricated timber frame elements. Taking into account a number cycles of erection and demolition design special attention was allocated to horizontal load distribution and spatial stiffening. Special plug connections and tensioning elements are used, combined with solvable screw connections. Temporary foundations complete the system.

Based on the modular conception the configuration of the modules can be adapted to the local requirements. Electrical- and media supply are also solved with plug-in connections.

The concept is not only realised as school replacement buildings, but as Mobi-Office also as office solutions, e.g. at TU Darmstadt. In between 25 projects were realized, three of them were demolished and erected again.

The Mobi-Space concept proofs, how modular and industrial construction is feasible even with high architectural quality.
\end{abstract}

Keywords room modules in timber construction system; reusability; serial building; temporary buildings; energy efficiency; building services; fire protection

Fast automatisiert werden dann Gegenargumente genannt: uniformes Bauen, fehlende Architekturqualität, hohe Transportkosten, geringe Flexibilität, nur geringe Preisunterschiede, mangelnde Integration von TGA usw. Bei diesen Diskussionen ist jedoch häufig festzustellen, dass die Protagonisten zunächst aneinander vorbeireden. Denn unter „modularem Bauen“ kann man sehr unterschiedliche Dinge verstehen. Ganz grundsätzlich ist „modulares Bauen“ ein Bauverfahren, bei dem ein Bauwerk in Gänze oder in Teilen aus vorgefertigten Bauteilen zusammengesetzt wird. Dies kann in Raumzellenbauweise erfolgen (3-D-Raummodule), zu der auch die Variante Containerbau gehört. Oder es werden Raummodule auf der Baustelle aus vorgefertigten Dach-, Wand- und Deckentafeln zusammengefügt. Beide Varianten können miteinander kombiniert werden. Theoretisch kann auch eine (Trag-)Skelettbauweise, ergänzt durch Dach-, De- 


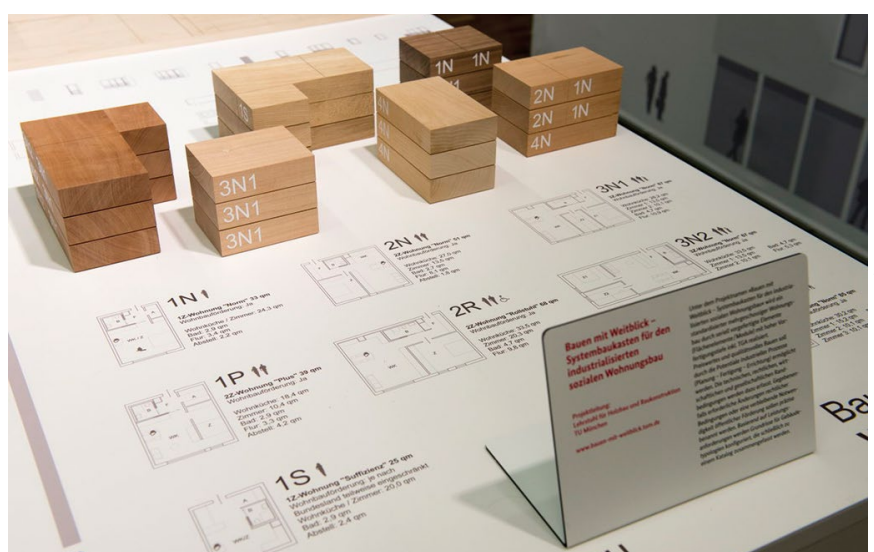

Bild 1 Modell zur Baugruppensystematik im Forschungsvorhaben „Bauen mit Weitblick"

Building constellation model created in research project „Bauen mit Weitblick"

cken- und nicht tragende Wandtafeln, als modulares Bauen bezeichnet werden; diese Variante wird im Folgenden nicht weiter betrachtet.

Ein Nachteil der derzeitigen Situation des modularen Bauens ist das Fehlen eines echten, produkt- und firmenübergreifenden Industriestandards zur Definition der Schnittstellen von Raummodulen und ergänzenden Bauteilen und häufig das mangelnde Interesse der Planer, mit einem klar strukturierten Baukasten individuelle Entwürfe $\mathrm{zu}$ entwickeln. In dem aktuell laufenden Forschungsprojekt „Bauen mit Weitblick“ [1] werden die Potenziale des modularen Bauens für den sozialen Wohnungsbau untersucht. In dem noch bis Ende des Jahres 2017 laufenden Projekt zeigt sich erneut, dass es zur Ausschöpfung der Vorteile modularen Bauens im Wesentlichen auf die klare Definition von Baugruppen ankommt, die in sich vollständig gelöst sind, eindeutig definierte Schnittstellen aufweisen und bei der Konfiguration zu Gebäuden keine weiteren Anpassungsplanungen mehr benötigen. Nur so kann eine tatsächliche Serienfertigung für dennoch individuelle Gebäude entstehen. Dabei gilt der Grundsatz: Eine Baugruppe sollte so groß wie nötig, aber so klein wie möglich sein. Die kleinste mögliche Baugruppe ist ein einzelnes Raummodul; zumindest für den sozialen Wohnungsbau zeigt sich aber, dass als eine Baugruppe mindestens eine Wohnung, häufig sogar eine stockwerksübergreifende Baugruppe mit mehreren Wohnungen zu definieren ist. Diese vollständig in sich gelösten Baugruppen können sowohl als 3-D-Raummodule als auch in Tafelbauweise produziert werden. Das Projekt zeigt auch, dass es zur Erzielung der angestrebten Preisvorteile von besonderer Bedeutung ist, für die in sich vollständig gelösten Baugruppen keine weiteren Anpassungsplanungen in den Unternehmen mehr vornehmen zu müssen. Eine Grundvoraussetzung für eine zumindest teilweise Serienfertigung.

\section{$2 \quad$ Das Bausystem Mobi-Space}

Seit Jahrzehnten gibt es zahlreiche Modulbaukonzepte im Holzbau. Ein jüngeres unter ihnen ist das Mobi-Space-

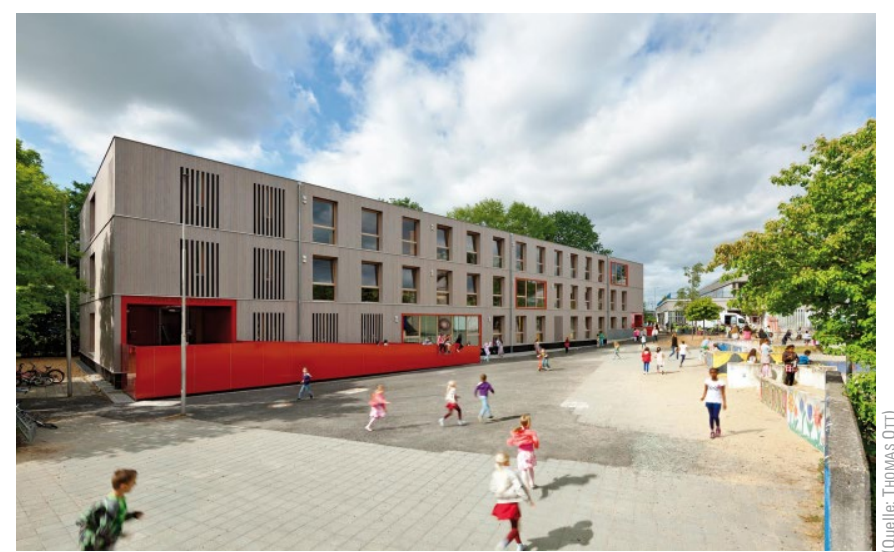

Bild 2 Mobi-Sku:I auf dem Gelände der Ludwig-Weber-Schule in FrankfurtSindlingen

Mobi-Sku:I at the area of Ludwig-Weber-school in Frankfurt-Sindlingen

System, das sich inzwischen mehrfach als Mobi-Sku:l, also als Schulersatzbau, aber auch als temporär geplanter Bürobau Mobi-Office bewährt hat.

Die Architekten des Darmstädter Büros werk.um haben mit ihren mobilen Mobi-Space-Gebäuden in modularer Holzrahmenbauweise gezeigt, dass es für spezielle Anwendungen grundsätzlich gelingen kann, die oben beschriebenen Anforderungen an modulares, serielles Bauen zu erfüllen.

Das Bausystem Mobi-Space wurde zunächst als MobiSku:l entwickelt, um während Grundsanierungen von Schulen den Schulgemeinden einen ansprechenden Ersatzbau mit deutlich anderen energetischen und räumlichen Qualitäten anzubieten, als sie bspw. reine Containerbauten anbieten können. Damit ist ergänzend die möglichst verschleißfreie Demontage des Gebäudes zu ermöglichen, um es nach erfolgter Grundsanierung des eigentlichen Schulgebäudes abbauen und an anderer Stelle bei der nächsten Sanierung wiederverwenden zu können (Bild 2).

Das erste, temporär aufgebaute Gebäude dieser Art war die dreigeschossige Mobi-Sku:l auf dem Gelände der zu sanierenden Georg-Christoph-Lichtenberg-Schule im hessischen Ober-Ramstadt. Der Landkreis Darmstadt-Dieburg ließ das Schulhaus 2011 für 2 Mio. Euro bauen. Mit 24 Klassenräumen diente es zwei Jahre lang 768 Schülern $(24 \times 32$ Schüler $)$ als Bleibe. In den Sommerferien 2013 wurde das temporäre Gebäude dann demontiert und in Weiterstadt - ebenfalls als Schulersatzbau - wieder aufgebaut, wo es bis heute steht (Bild 3).

Da die Entwicklung eines solchen Systems wegen des derzeit fehlenden Industriestandards nur im Team mit einer ausführenden Firma und weiteren Planern gelingen kann, wurde die Entwicklung zusammen mit den Firmen Baumgarten aus Ebersburg/Weyhers (Holzbau) und der bauart Konstruktions Gesellschaft aus Lauterbach (Tragwerksplanung, Bauphysik, Brandschutz) betrieben. 


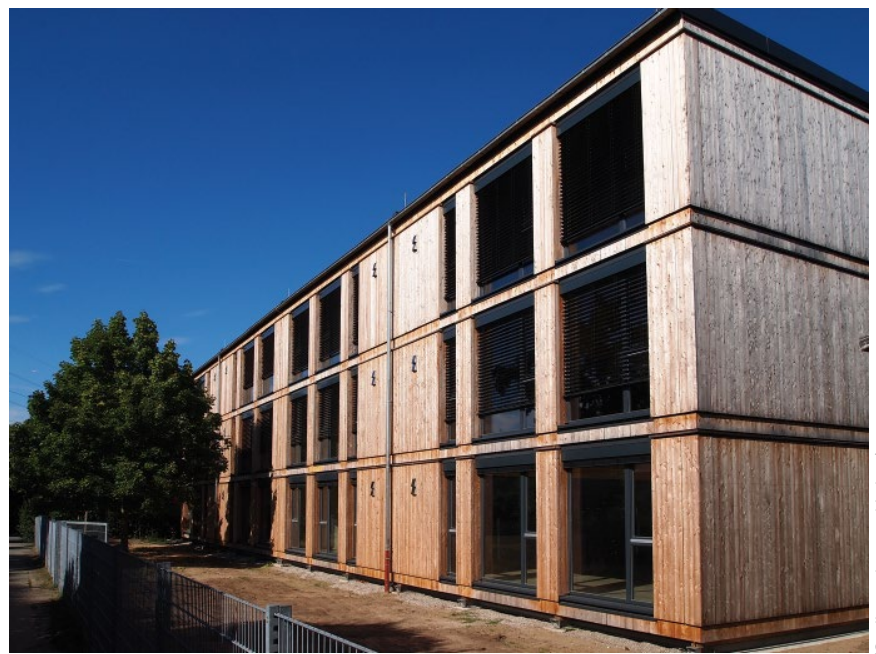

Bild 3 Wiederaufbau als Ersatzbau an der Albrecht-Dürer-Schule in Weiterstadt

Re-erected Mobi-Sku:l at Albrecht-Dürer-school in Weiterstadt

\section{3}

\section{Anforderungen an das System Mobi-Space}

Die wesentlichen Anforderungen an das System sind eine schnelle Errichtung, gute Demontier- und Wiederverwendbarkeit, ein energetischer Standard mindestens auf, besser oberhalb des EnEV-Niveaus und in den dreigeschossigen Gebäuden ein den regulatorischen Anforderungen entsprechender Schall- und Brandschutz. Dazu kommen Anforderungen an die Lüftung, variable Energieversorgungsmöglichkeiten und optimierte Transportmaße und Hebegewichte. Selbstverständlich ist die erforderliche Einhaltung des Sicherheitsniveaus in der Tragwerksplanung. Es können keine Teilsicherheiten reduziert oder auf eine Baugenehmigung verzichtet werden, da es sich nicht um „temporäre“ Bauten (fliegende Bauten) handelt. Nach den Bauordnungen der Länder sind sogenannte fliegende Bauten zwar bauliche Anlagen, „die geeignet und bestimmt sind, wiederholt aufgebaut und zerlegt zu werden“, ihre Standzeit ist aber i.d. R. auf drei Monate beschränkt. Bei den hier aus Modulen errichteten Gebäuden sind aus Gründen der Nutzungssicherheit und des Nutzungskomforts alle eingeführten technischen Baubestimmungen einzuhalten und alle baurechtlichen Verwendbarkeitsnachweise uneingeschränkt zu erbringen.

Für den Schulbau wurde zunächst ein Baukastensystem aus $54-\mathrm{m}^{2}$-Modulen entwickelt.

Die Bauart ist eine Holztafelbauweise. Die vorgefertigten Wand- und Decken- bzw. Bodenelemente sind so konzipiert, dass ein mehrmaliger Auf- und Abbau möglich ist. Die daraus zusammengesetzten Raummodule mit einer Fläche von $54 \mathrm{~m}^{2}(b / l=6 \mathrm{~m} \times 9 \mathrm{~m})$ können beliebig addiert und bis zu drei Geschossen gestapelt werden. Diese Konzeption entspricht der oben beschriebenen Baugruppen-Systematik.

Jeder Pavillon besteht aus zehn Teilen: drei Boden-, drei Decken- und vier Wandelemente. Ihre Abmessungen

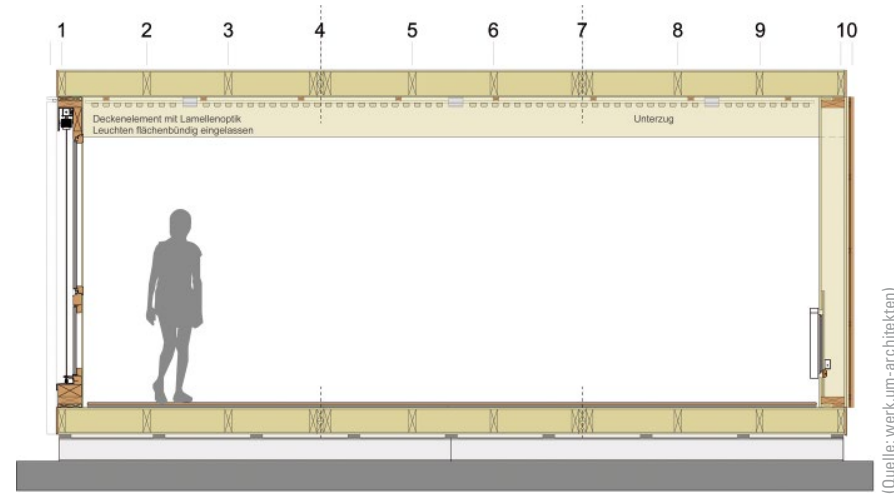

Bild 4 Mobi-Space Grundmodul Mobi-Space basic module
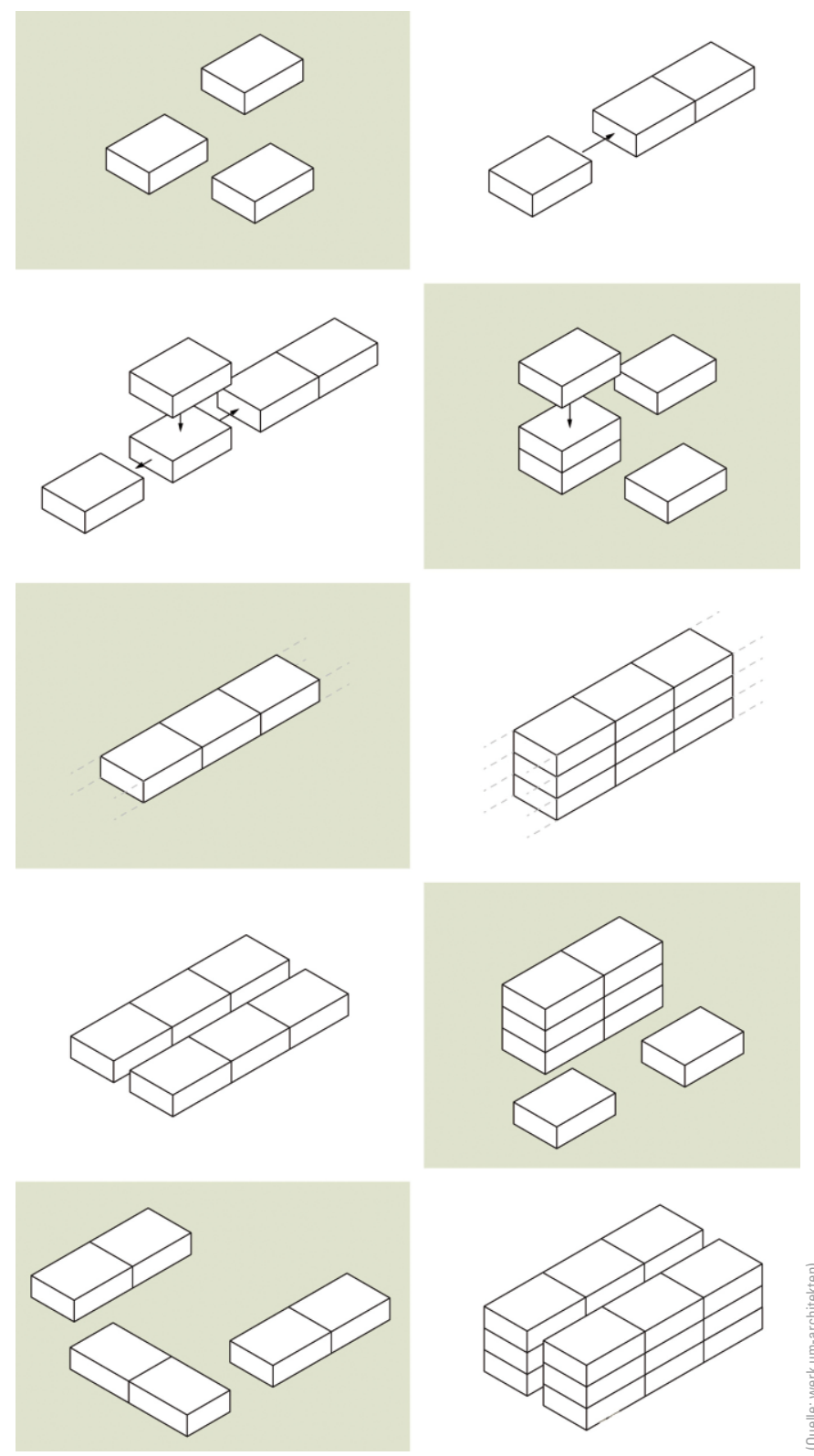

Bild 5 Mobi-Space - mögliche Konfigurationen Mobi-Space - types of configuration

sowie die Bauteildicken sind bei allen Mobi-SpaceBauten standardisiert, das Gebäuderaster ist mit 2,91 m/3,01 m/2,91 m auf einfache Montage und Trans- 
port abgestimmt. Die $6 \mathrm{~m}$ und 9,43 $\mathrm{m}$ langen Wandelemente haben eine Höhe von 2,65 m. Zwischen die Stiele der Holztafelkonstruktion sind $200 \mathrm{~mm}$ ZellulosefaserWärmedämmung eingebracht, die Tafelelemente sind beidseitig mit $15 \mathrm{~mm}$ dicken OSB-Platten beplankt.

Auf der Fassadenseite sind auf einer diffusionsoffenen Holzwerkstoffplatte Lattung und Konterlattung und eine Fassadenbekleidung aus $20 \mathrm{~mm}$ Lärchenbrettern aufgebracht. Die Wände der Gebäudehülle erreichen in der Summe eine Dicke von etwa 300 mm.

\section{4}

\section{Technische Lösungen}

Das Modulsystem Mobi-Space ist für Gebäude bis zu drei Geschossen ausgelegt. Auf eine lastbedingte Staffelung der Bauteilabmessungen wurde zugunsten einer einfachen Montage verzichtet. Alle Holztafelbauelemente und Unterzüge eines Moduls sind gleich dimensioniert und können in jedem Geschoss eingebaut werden. Maßgebend für die Dimensionierung sind somit die Beanspruchungen eines EG-Moduls in einem dreigeschossigen Gebäude im EG. Die Bauteile im 1. und 2. OG sind entsprechend überdimensioniert. Dieses Vorgehen ist typisch, wenn man eine tatsächliche Serienfertigung erreichen will, in diesem Fall tritt die Ressourcen-Effizienz gegenüber der Rationalisierung von Planung und Herstellung zurück. Dieses Vorgehen widerstrebt so manchem Ingenieur, ist aber im Sinne industriellen Bauens wohl unumgänglich. Der Holzbau tröstet dabei wenigstens mit einer erweiterten Kohlenstoffspeicherung und damit einer verbesserten $\mathrm{CO}_{2}$-Senkenfunktion!

Die Decken- und Bodenelemente sind ebenso gleichartig ausgelegt und durchgehend für $3 \mathrm{kN} / \mathrm{m}^{2}$ Nutzlast nachgewiesen. Sie können gleichzeitig mit entsprechender außen aufgebrachter Wärmedämmung als Dachelemente verwendet werden.

Die Spannweite der Raummodule ist mit $6 \mathrm{~m}$ Breite relativ groß und mit Holztafelbauelementen weder konstruktiv noch wirtschaftlich sinnvoll zu überbrücken. In Querrichtung verlaufen daher je Raummodul zwei Brettschicht-Holz-Unterzüge $(b / h=200 \mathrm{~mm} \times 400 \mathrm{~mm})$. Die Spannweite der Deckenelemente wird damit auf $3 \mathrm{~m}$ reduziert - was auch ein besseres Transportmaß ist - und die Konstruktionshöhe der Decken- bzw. Dachelemente entspricht mit $200 \mathrm{~mm}$ Dicke der Wandkonstruktion (einheitliches Material/Lagerhaltung).

Die bis zu den Längswandaußenkanten geführten Decken messen 6,43 $\mathrm{m}$ in der Länge, sind aber unterschiedlich breit: Die beiden Randelemente spannen mit 3,21 m von den Außenkanten der kurzen Raummodulwände bis zur Achse des ersten Unterzugs und das Mittelteil mit $3 \mathrm{~m}$ von Unterzugachse zu Unterzugachse.

Außenwandaufbau und Innenwand bzw. Flurwand sind aufeinander abgestimmt, sodass auch hier ein Austausch

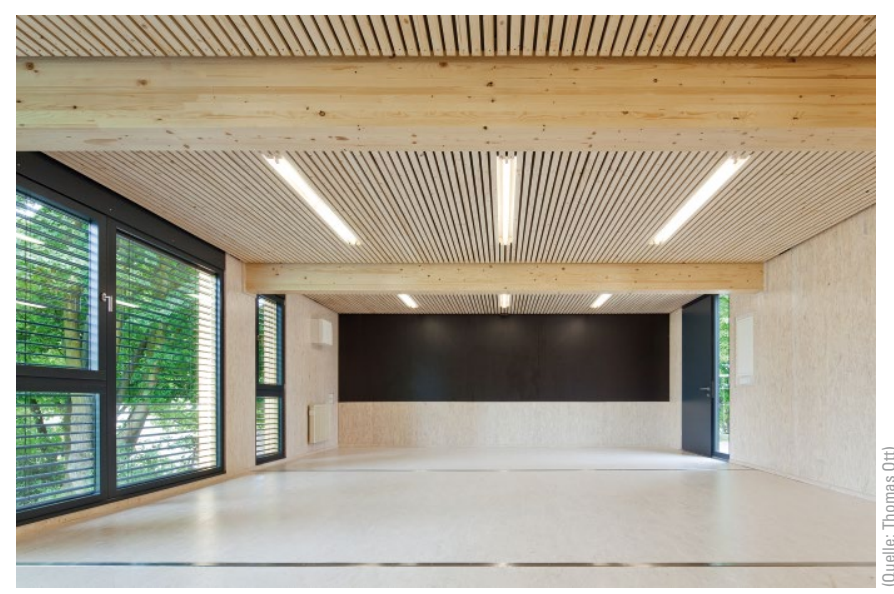

Bild 6 Innenraumansicht eines Klassenzimmers Interior view of a class room

bzw. Umbau mit minimiertem Aufwand möglich ist. Die Fundamente werden standortspezifisch konstruiert, bemessen und ausgeführt.

Aus der geforderten Demontierbarkeit und Remontierbarkeit ergeben sich einige weitere Besonderheiten für die Tragwerksplanung hinsichtlich der konstruktiven Durchbildung, z. B. keine Verwendung von Nagel- oder Klammerverbindungen, sondern vorzugsweise Bolzenverbindungen (Bild 8), ggf. Voll- oder Teilgewindeschrauben. Letztere bergen aber das Risiko, bei Herausschrauben nach längerer Standzeit abzureißen - mit dem daraus folgenden Aufwand für den Ausbau. Zur horizontalen Kraftübertragung wurden ursprünglich Eichendollen als Schubverbinder (Bilder $7,8)$ verwendet, die gleichzeitig Montagehilfe waren. Im Zuge der Überarbeitung/Weiterentwicklung (2016) sind diese jedoch durch Bolzenverbindungen mit oberseitiger Stahlplatte und unterseitiger Scheibenmutter bei den Anschlüssen Decke-Wand ersetzt und bei den Verbindungen Fußboden-Wand ergänzt worden.

Das Konstruktionsprinzip entspricht der Plattformbauweise, die bei bis zu dreigeschossigen Gebäuden gut anwendbar ist. Die aus horizontalen Beanspruchungen entstehenden vertikalen Zugkräfte werden über vertikale Verbindungen übertragen, die in der Ausführung sinngemäß der in Bild 8 gezeigten Verbindung entsprechen. Die Verbindungen sind für den schnellen Auf- und Abbau sehr gut geeignet. Sie lassen sich bei der Demontage leicht lösen, die Wand- und Deckenelemente können einfach wieder ausgebaut werden.

Zwischenzeitlich wurde die Kopplung und Aneinanderreihung (zunächst in einer Ebene; EG) weiterentwickelt, um die freistehende Errichtung eines Raummoduls oder von zwei Raummodulen mit dazwischengeschaltetem Flur zu ermöglichen. Die Elemente selbst bleiben gleich und die erforderlichen Anpassungen werden auf ein Minimalmaß reduziert; d.h., ein Bauherr kann frei werdende Anlagen an neuem Standort neu miteinander kombinieren. „Beliebige“ Mehrfachaufstellungen werden ermöglicht. Auch hier wird versucht, Entwicklungsschritte 


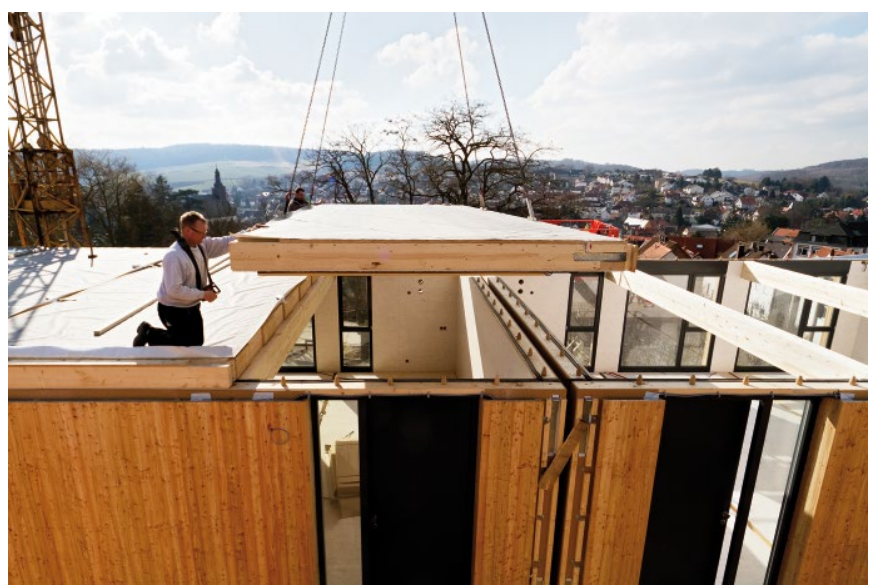

Bild 7 Verlegung eines Deckenelements auf einem Raummodul Installation of a ceiling panel on top of a basic module

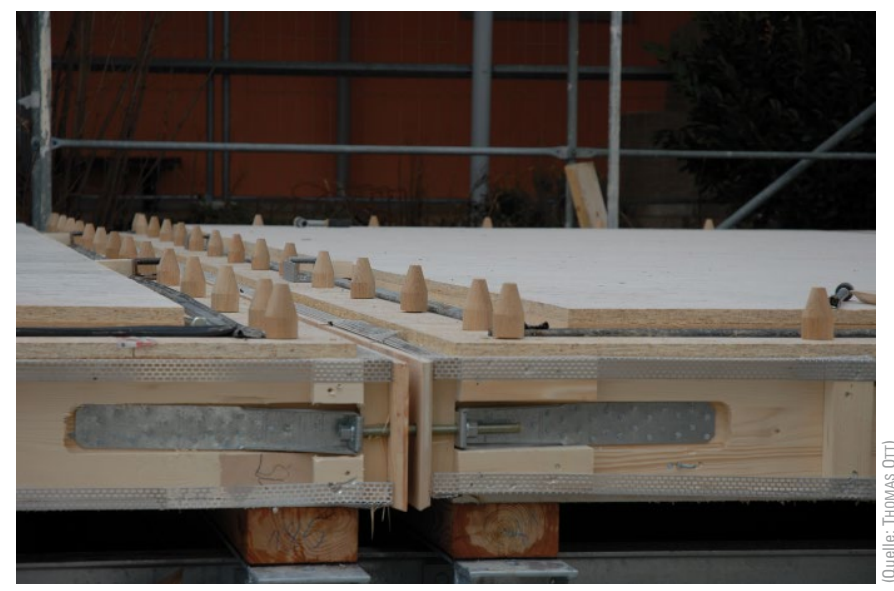

Bild 8 Hartholz-Schubverbinder und Zugverbindung zur Ausbildung des Zuggurts einer Deckenscheibe

Hardwood shear-connector an tension connection to realise a the tension girder of a ceiling plate

kann vorgefertigt werden, wie hoch ist der abschließende Ergänzungsaufwand auf der Baustelle?

Die Stromversorgung kann über einfache, standardisierte Steckverbindungen erfolgen. Bei der Mobi-Sku:l wurden die Elemente/Pavillons lediglich mit Steckern verbunden und so an die Strom- und Medienversorgung angeschlossen. Licht und Lüftung sind somit schnell erledigt. Ein besonderer Vorteil besteht dann, wenn eine elektrische Direktheizung über Flächenelemente verwendet wird. Der relativ aufwändige Anschluss an ein Nah- oder Fernwärmenetz oder die Errichtung einer eigenen kleinen Heizzentrale, die Verlegung und Kopplung von Heizungsvor- und -rücklauf sowie Wartungskosten entfallen. Bei der Untersuchung der Verwendung von Strom-Direktheizungen im vorgenannten Forschungsprojekt „Bauen mit Weitblick“ [1] konnte ermittelt werden, dass bei entsprechendem energetischen Standard des Gesamtgebäudes, dem Einsatz von Fotovoltaik und einer Abluftwärmepumpe mit Wärmerückgewinnung für die Warmwasserversorgung, der Nachweis des Primärenergiebedarfs selbst nach ENEV 2014 bereits gelingt. Der sich durch den Ausbau erneuerbarer technische Gebäudeausrüstung: Was ist erforderlich, was
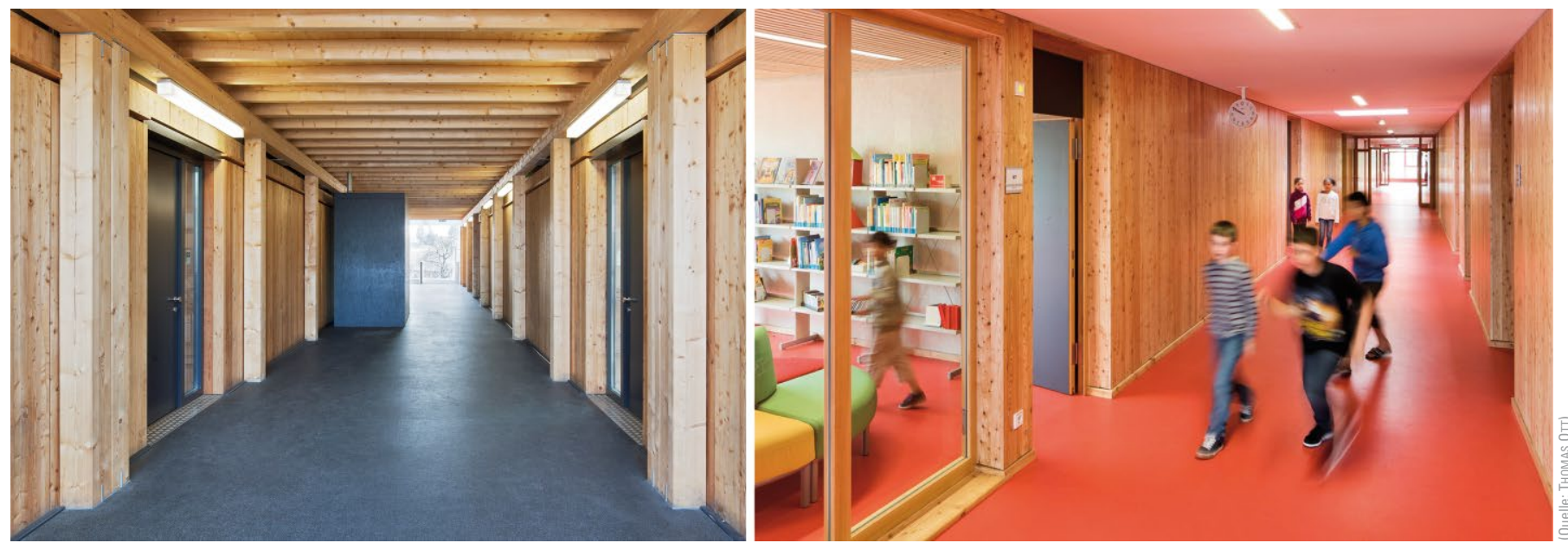

Bild 9 Flur des Mobi-Space mit Stützen-Träger-Konstruktion (I.), Flur der Mobi-Sku:I ohne Stützen-Träger-Konstruktion (r.) Corridor area of Mobi-space with a beam-column-structure (I.), corridor area of Mobi-Sku:I without an additional structure (r.) 


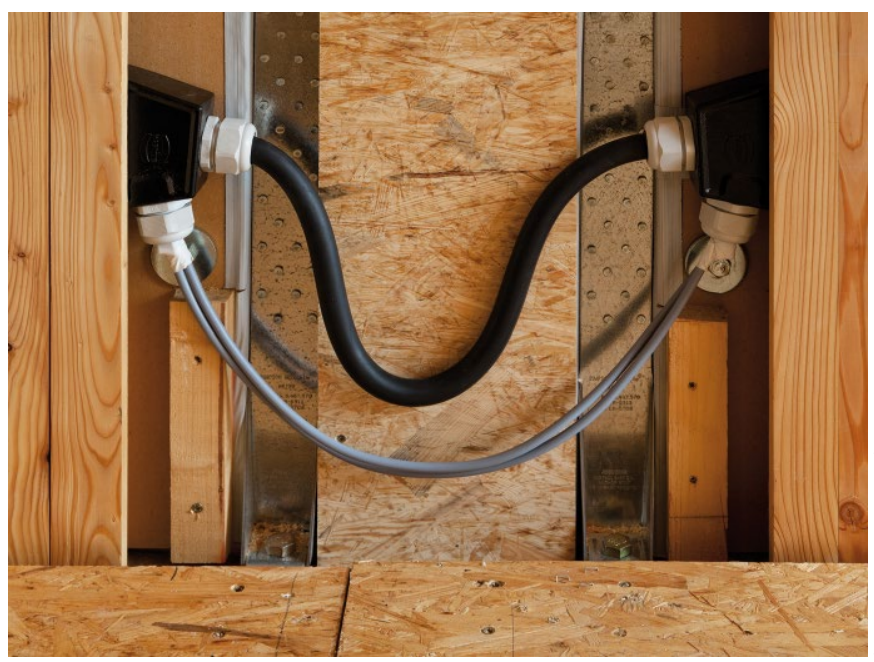

Bild 10 Kopplungsstelle für Strom zwischen zwei Raummodulen vor Zugverbindungen zur vertikalen Zugverankerung

Electrical connection of two basic modules in front of two vertical tension connections of vertical anchoring

Energien weiter verändernde Strommix unterstützt diese Tendenz.

Die Entwicklung von Mobi-Space ist eine „spezifische“, firmenbezogene Variante des Modulbaus. Eine enge und direkte Abstimmung zwischen der Tragwerksplanung und Konstruktionsabteilung des ausführenden Unternehmens führt zu optimierten Bauteilen, die nach Konfiguration des Gebäudes durch die Architekten keine weitere Anpassungsplanung der Raummodule selbst mehr benötigen. Ein wesentlicher Kosten- und Zeitaspekt.

\section{Unvermeidbare Anpassungen}

Trotz aller Kombinationsmöglichkeiten sind geringfügige Anpassungen im Gesamtgebäude manchmal unvermeidlich, z.B. aus Bebauungsplänen resultierende Gebäudebreiten. Bei frühzeitiger Abstimmung zwischen Bauherrn und Bauteam sind solche individuellen Lösungen - z.B. breitere Flure, zusätzliche Treppen etc. - ohne nennenswerten Mehraufwand möglich.

Dass die Fundamentierung je nach Standort angepasst werden muss, ist ebenso unvermeidlich.

Als Fundament für die Modulbauten wird üblicherweise ein Stahlträgerrost auf Einzelfundamenten verwendet, eventuell ergänzt oder ersetzt durch Streifenfundamente. Eingeschossige Bauten kommen auch mit Punktfundamenten oder Schraubgründungen aus maschinell eingedrehten Erdschrauben aus.

Die Wahl der Gründung erfolgt immer in Abhängigkeit von der Bodenbeschaffenheit und unter Berücksichtigung vorhandener Leitungstrassen unter dem temporären Bauplatz. Auf dem Trägerrost werden dann die Schwellhölzer verlegt, darauf folgen die Bodenelemente.
Auch der sommerliche Wärmeschutz muss (bedingt durch die unterschiedliche Ausrichtung der Grundstücke) angepasst werden, zusätzliche außen liegende Verschattungen können erforderlich werden. Der Energiestandard der Mobi-Space-Gebäude in heutiger Ausführung übertrifft die Anforderungen zur Gebäudehülle der EnEV 2009 und erfüllt heute die EnEV 2014.

Abschließend sind ggf. die brandschutztechnischen Maßnahmen anzupassen. Durch die Beschränkung auf bis zu dreigeschossige Gebäude und damit auf Gebäude geringer Höhe (GK 3 nach MBO 2008) genügt für den Feuerwiderstand der Bauteile eine feuerhemmende Ausführung (F30-B), die durchgehend nachgewiesen ist. Bezogen auf die jeweilige Konfiguration sind jedoch die Rettungsweglängen und -breiten sowie die Anordnung und Zahl der Treppenhäuser festzulegen. Als sinnvolle Kombination haben sich für die Mobi-Sku:l bisher 24 Klassen in drei Geschossen mit zwei unabhängigen Treppenanlagen erwiesen. Diese sind im Regelfall als außen liegende Stahltreppen konzipiert, müssen aber zum Teil trotzdem mit Rauchschutztüren von den unbeheizten, als Außenraum geltenden Fluren abgetrennt werden. Die genauen Festlegungen, z.B. zu einer gesonderten Brandabschnittsbildung oder zur Einhaltung der Abstandsflächen, sind der Bewertung in einem objektbezogenen Brandschutzkonzept überlassen. Entscheidend ist auch hier, dass Anpassungen wie z.B. ein innen liegendes, beheiztes Treppenhaus im System und über festgelegte Schnittstellen erfolgen.

\section{$6 \quad$ Kostengünstiges Bauen erfordert Kompromisse}

In diesen Gebäuden einen „erhöhten Schallschutz“ im Sinne der DIN 4109:1989-11, Beiblatt 2 realisieren zu wollen, gehört z.B. zu diesen Einschränkungen. In den Schulersatzbauten wird derzeit ein „Trockenestrich“ aus 22 mm OSB-Platte auf Trittschalldämmung verlegt, unterseitig der Holztafelelemente ist eine Akustikdecke montiert. Diese Ausführung führt bisher zu einer hinreichenden Akzeptanz durch die Nutzer, genaue Messwerte dazu liegen nicht vor. Wohlgemerkt: Dies gilt für die temporären Gebäude, die jedenfalls einfach rückbaubar sein müssen! Bei dauerhafter Nutzung sind die Bodenaufbauten so zu modifizieren, dass die Anforderungen der DIN 4109:2016 für den normalen Schallschutz erfüllt werden. Entsprechende Raumhöhen sind dazu verfügbar. Bezüglich der Schalldämmung der Wände sind die Trennwände zwischen den Klassenräumen wegen der doppelschaligen Ausführung unproblematisch, die Flur- und Außenwände erfüllen die bauaufsichtlichen Vorgaben.

Auch bei der Lüftung hat man sich zur Einfachheit entschlossen: In jedem Raum gibt es eine dezentrale Lüftungsanlage mit Wärmerückgewinnung. Sie ermöglicht eine Grundlüftung/Luftwechselrate von ca. $0,11 \mathrm{~h}^{-1}$, was für bis zu 30 Schüler im Betrieb nicht ausreicht. Es muss zusätzlich regelmäßig über die Fenster gelüftet werden. Die Lüftungsanlage dient vor allem der Grundlüftung, 
damit die Räume morgens zu Unterrichtsbeginn mit Frischluft versorgt sind.

Diese Einschränkungen haben nichts mit der modularen Bauweise zu tun, sie beziehen sich vielmehr auf ergänzende Aspekte kostengünstigen Bauens und auf die Anforderung der Umsetzbarkeit.

\section{$7 \quad$ Bauzeit und Ausschreibung}

Nach Kran- und Gerüststellung sowie Montage der Unterkonstruktion lässt sich ein dreigeschossiges MobiSpace-Gebäude - ob Sku:l, Office oder etwas ganz anderes - in $4 \mathrm{~d}$ errichten.

Die Elemente des Baukastensystems sind außer in ihren Bauteilabmessungen auch sonst für einen mehrfachen Auf- und Abbau sowie Transport ausgelegt. So sind beispielsweise die Steckdosen zurückversetzt eingebaut und damit geschützt, ebenso die Lichtschalter und Leuchten.

Die vorgefertigten Wand-, Decken- und Dachelemente erhalten Fenster, Türen, Sonnenschutz, Beleuchtung, Heizung, kontrollierte Lüftung und Steckdosen bereits werkseitig.

Bei der Mobi-Sku:l wurden die Raummodule lediglich mit einem Stecker verbunden und so an die Strom- und Medienversorgung angeschlossen (Heizung elektrisch). Weitere acht Wochen Bauzeit sind nach der Montage für die Baufertigstellung, den Innenausbau zu kalkulieren. Dann sind die Räume bezugsfertig.

\section{Literatur}

[1] Bauen mit Weitblick. Verbund-Forschungsprojekt, TU München, Fraunhofer Institut für Bauphysik, Fa. Max Bögl, Fa. Regnauer Fertigbau und KoWo mbH Erfurt, Bonn: Forschungsinitiative Zukunft Bau, Bundesamt für Bauwesen und Raumordnung, Deichmanns Aue 31-37, 2016-2017 (laufend).
Modulare Bauweisen sind eigentlich sinnvoll nur funktional auszuschreiben. Idealerweise erfolgt ggf. in einem zweistufigen Verfahren die Vergabe an einen Generalunternehmer, was hier vorzugsweise das Holzbauunternehmen ist. Eine getrennte Vergabe für Fundamentierung, Erdarbeiten und Außenanlage erscheint als gerade noch sinnvoll, alle anderen Gewerke müssen gebündelt bleiben. Die Position „Aufbau, Abbau, Transport und Wiederaufbau an anderer Stelle im Umkreis von $25 \mathrm{~km}$ " ist ebenfalls als eine Eventualposition $\mathrm{zu}$ inkludieren. Aus haftungsrechtlichen Gründen erscheint es nur sinnvoll, dass das ausführende Holzbauunternehmen auch letztgenannte Position übernimmt.

\section{Ausblick}

Das Konzept wurde bisher nicht nur als temporärer Schulersatzbau, sondern auch als Mobi-Office, z.B. an der TU Darmstadt, realisiert. Insgesamt wurden bisher 25 Projekte errichtet, drei davon wurden bereits wieder abgebaut und an einem neuen Standort errichtet.

Das Konzept zeigt beispielhaft, welche Randbedingungen für serielles Bauen erforderlich sind und dass eine Bewegung hin zu einer echten Industrialisierung und Standardisierung auch mit sehr guten architektonischen Leistungen verbunden sein kann. Denn ob die Mobi-Space-Bauten in allen Fällen „temporär“ bleiben, steht offen: Wegen der sehr ansprechenden Optik und des angenehmen Raumklimas würden einige Nutzer ihre Gebäude am liebsten behalten.

\author{
Autoren \\ Univ.-Prof. Dr.-Ing. Stefan Winter \\ Technische Universität München \\ Lehrstuhl für Holzbau und Baukonstruktion \\ Arcisstraße 21 \\ 80333 München \\ und \\ bauart Konstruktions GmbH \& Co. KG \\ Beratende Ingenieure \\ Spessartstr. 13 \\ 36341 Lauterbach \\ winter@bauart-konstruktion.de \\ Dipl.-Ing. (FH) Susanne Jacob-Freitag \\ manuScriptur \\ Freie Journalistin (DJV) \\ Schubertstraße 21 \\ 76185 Karlsruhe \\ info@texte-nach-mass.de \\ Claudia Köhler M.Sc. \\ bauart Konstruktions GmbH \& Co. KG \\ Beratende Ingenieure \\ Destouchesstraße 65 \\ 80796 München \\ koehler@bauart-konstruktion.de
}

